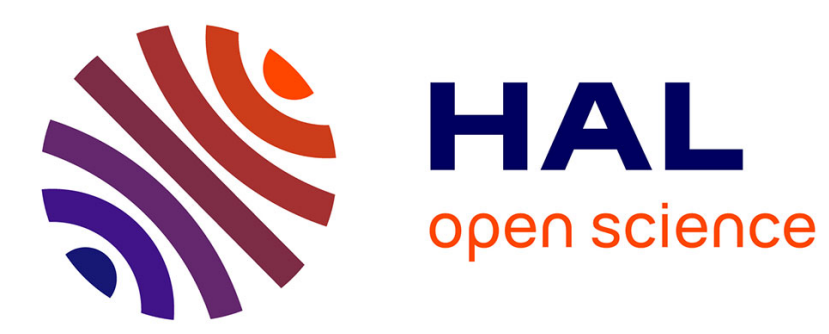

\title{
Sur la luminescence résiduelle dans l'infra rouge des cristaux et microcristaux photoluminescents \\ F. Martin
}

\section{To cite this version:}

F. Martin. Sur la luminescence résiduelle dans l'infra rouge des cristaux et microcristaux photoluminescents. J. Phys. Radium, 1930, 1 (11), pp.401-403. 10.1051/jphysrad:01930001011040100 . jpa-00233039

\section{HAL Id: jpa-00233039 https://hal.science/jpa-00233039}

Submitted on 1 Jan 1930

HAL is a multi-disciplinary open access archive for the deposit and dissemination of scientific research documents, whether they are published or not. The documents may come from teaching and research institutions in France or abroad, or from public or private research centers.
L'archive ouverte pluridisciplinaire HAL, est destinée au dépôt et à la diffusion de documents scientifiques de niveau recherche, publiés ou non, émanant des établissements d'enseignement et de recherche français ou étrangers, des laboratoires publics ou privés. 


\title{
SUR LA LUMINESCENGE RÉSIDUELLE DANS L'INFRA ROUGE DES CRISTAUX ET MIGROGRISTAUX PHOTOLUMINESGENTS
}

\author{
par F. MARTIN
}

\begin{abstract}
Sommaire. - La luminescence résiduelle des cristaux et microcristaux photoluminescents signalée par plusieurs auteurs a été étudiée dans l"infra-rouge du filtre optique de Charbonneau de même que l'illumination fugace et l'effet extincteur Becquerel.

La méthode expérimentale consiste à exciter en lumière de Wood et à détecter au moyen d'une cellule photoélectrique connectée à l'amplificateur différentiel à triodes de Lévy et Rio, les phénomènes étant observés en fonction do la persistance décroissante de la phosphorescence.

L'ensemble des faits établis montre une relation entre la disparition de l'illuminalion fugace, de la luminescence résiduelle ainsi que de l'effet extincteur et la durée de réémis. sion de la lumière.
\end{abstract}

Généralités et Bibliographie. - L'apparition dans le spectre visible de la phos. phorescence latente sous l'action d'un faisceau de rayons infra-rouges, présentée par certaines substances cristallines ou microcristallines photoluminescentes, est un des plus curieux phénomènes qui s'offre à l'attention du physicien dans le domaine de la lumière froide. Le iait que l'on puisse faire surgir des radiations visibles d'un corps obscur en l'irradiant dans l'obscurité la plus complète par des rayons obscurs infra-rouges, paraît au , premier abord paradoxal et en opposition formelle avec la règle de Stokes qui constitue la loi fondamentale de la phosphorescence.

G. Le Bon $\left(^{1}\right)$ avait déjà établi que les sulfures de calcium et de strontium photoluminescents conservent après leur extinction normale une luminescence rémanente qu'un peut rendre visible en projetanl des rayons infra-rouge à leur surface. J. Risler $\left(^{2}\right)$ a constaté également qu'une statuette enduite de sulfure de calcium préalablement excité, redevenue obscure et conservée dans l'obscurité complète, fait apparaittre quand on la soumet au rayonnement infra-rouge « une luminosité étrange suffisante pour impressionner la plaque photographique ". A Guntz $\left({ }^{3}\right)$ a découvert que le sulfure de zinc qui doit sa luminescence jaune orangé à une trace de manganèse, conserve plusieurs jours après l'excitation une luminescence latente qui réapparait brillamment dans un faisceau infra-rouge, et cet auteur a utilisé ce phénomène dans sa méthode de pholographie infra-rouge par phosphorographie. Nous ( ${ }^{4}$ ) avons enfin étudié dans les principaux domaines du spectre la variation en fonction du temps du seuil de thermoluminescence résiduelle des cristaux.

Méthode expérimentale et appareillage. - Les cristaux et microcristaux photoluminescents conservés pendant six mois dans l'obscurité pour éliminer les effets résiduels antérieurs sont excités par une lampe à vapeur's de mercure brûleur quartz type $\mathbf{N}$ L Gallois

(1) G. Le Bov. L'Evolution des forces [livre IV, chap. I, (1910). p. 287-292]. - La luminescence invisible [Revue scientifique, 28 janvier 1899].

$\left({ }^{2}\right)$ J. Rislkr. Tubes fluorescents et radiations excitant la fluorescence [Bull. scienl. des Eludiants de Paris juin 1923, p. 20],

(3) A. Gormz. La photographie infra-rouge par phosphorescence [IV॰ Congrès chimie industrielle (1925). septembre 1923, p. 2. - Photographie infra-rouge par phosphorographie |Comm. Soc. Phys. section Nancy, 10 mars 1924. Bull. Soc. fr. Phys., p. 7.35-763, (mai 1924).]

(4) F. Martis. Contribution à l'étude de la luminescence invisible dans les cristaux thermoluminescents [Congrès Soc. Savantes, Alger, 1930]. 
conjuguée au filtre Wood à base d'oxyde de nickel, laissant passer la bande $3663 \AA$ à $3650 \AA$ et la raie 3341 i arec une transmission maximum pour 3630 i. Pour les grandes longueurs d'onde, on a employé comme source une $600 \mathrm{~b}$. tungstène-azote avec, dans le rouge visible et le début du proche infra-rouge, les écrans Wratten 88, 89 et $89 \mathrm{~A}$ ainsi que le verre rouge d'Téna et le flint D. 262 Para Mantois qui sous une épaisseur de $\mathbf{1 0 , 6} \mathbf{m m}$ laisse passer plus de 90 pour 100 des radiations de longueur d'onde inférieures à $2,14 \mu$. Dans le proche infra-rouge qui a été surtout utilisé, le filtre optique était constitué par un verre noir de Charbonneau obscurci aux oxydes de manganèse et de cuivre fournissent entre $0,7 \ddot{\partial} \mu_{\text {e et }} 4,8 \mu$ une transmission infra-rouge qui atteint 85 pour 100 dans la raie correspondant à $2, \breve{s} ; \mu$.

La luminescence résiduelle a été observée au moyen de l'œil sensibilisé par un stage d'obscuration de trente minutes et contrôlée par une cellule photoélectrique au potassium dans argon avec amplificateur différentiel à triołes Lévy et Rio. Les plages luminescentes étaient préparées en coulant sur des plaques de verre les substances étudiées diluées dans le vernis amylaq we (éther acétique, benzine, 4,5 pour 100 celluloïd).

Résultats expérimentaux. - Lne première série de recherches a porté sur de nombreux sulfures phololuminescents constitués surtout par des diluants alcalino-terreux et ceux de la famille du zinc sensibilisés par les phosphorogènes cuivre, manganèse, bismuth, etc. En particulier $\mathrm{CaS}(\mathrm{Bi}, \mathrm{Na})$ à luminescence violette, $\mathrm{SrS}(\mathrm{Bi})$ à phosphorescence bleue, ZnS-CdS 12 pour $100(\mathrm{Cu})$ jaune citron, ZnS-CdS 20 pour 100 (Cu) orangé, ZnS-Cd 23 pour $100(\mathrm{Cu})$ rouge, $\mathrm{ZnS}(\mathrm{Mn})$ rouge, $\mathrm{ZnS}(\mathrm{Cu}$, Na) rert, et le sulfure de magnésium à luminescence bleuâtre ont été spécialement étudiés. L'irradiation par la lampe à vapeur de mercure était faite de manière à utiliser au maximum leur spectre d'excitation $(3 \check{000} \AA$ à 5300 à pour la bande d'émission $\alpha$ du cuivre avec $Z$ nC-Cd \& 12 pour $100(\mathrm{Cu})$. (A. Guntz).

Nous avons constaté que dans ces conditions, presque tous ces corps présentent le phénomène de la luminescence résiluelle avec une intensité très variable d'ailleurs; maximum avec le phosphorogène manganèse, elle devient extrèmement faible dans le cas de $\mathrm{ZnS}(\mathrm{Cu} \mathrm{Na})$ à luminescence verte et même nulle dans certains échantillons de ce sulfure. Tous font apparaitre nettement dans l'infra-rouge de Charbonneau et mieux encore dans le rouge visible l'effet extincteur Becquerel précédé de l'illumination fugace. Avec les sulfures photoluminescents à persistance courte comme ceux à luminescence rouge on obtient lorsque l'extinction normale est presque complète tous les intermédiaires entre l'illumination fugace et l'apparition de la luminescence latente suivie elle aussi d'un effet extincteur Becquerel.

Si l'on passe de ces substances microcristallines au groupe des cristaux naturels pour lesquels la persistance lumineuse se réduit à l'ordre de la minute, on observe après excitation sans filtre Wood afin d'augmenter la luminosité, un effet extincteur qui dans le rouge visible apparait sur de nombreuses variétés de chlorophanes : Etressin, Royat, Pont du Château, Saugeac, Chavagnac-Lafayette, Roumiga ainsi qu'avec la fluorine violette de Durham, la fluorine jaune de Cumberland, l'apatite de Renfrew et la leucophane de Brewig. Cet effet extincteur est beaucoup plus faible et mème souvent absent dans l'infrarouge de Charbonneau ainsi que l'avait déjà observé Maurice Curie; la luminescence résiduelle qui devient moins nette est cependant encore visible avec la fluorine violette de Durham.

En poursuivant cetle étude parmi les cristaux photoluminescents où la persistance tombe à l'ordre (le la seconde et nécessite dans la plupart des cas l'emploi du phosphoroscope on voit disparaître simultanément la luminescence résiduelle l'illumination fugace et l'effet extincteur. En effet les résultats ont été négatifs avec la calcite d'Auvergne, l'aragonite, la withérite, la barytine, la strontianite, la kunzite (variété triphane) de Minas Geraes, la cryolite d'Irikaet, le disthène de Suisse, le tungstate de calcium, les fluorines transparentes des Pyrénées et du Puy-de-Dòme, etc.

Enfin si l'on atteint avec les cristaux fluorescents une durée de réémission de lumière 
qui devient extrêmement courte et de l'ordre de 10-> sec. (L. Gaviola. (') F. Perrin $\left(^{2}\right)$ ) on observe les faits suivants avec une excitation simultanće en lumière de Wood et avec linfra-rouge de Charbonneau suivant la méthode de Maurice Curie ${ }^{3}$.

La fluorine violette de Durham, les chlorophanes de Saugeac, Pont du Chateau, Chavagnac-Lafayette qui présentent dans l'ultra-violet de Wood de magnifiques fluorescences violettes ne montrent ni dans le rouge visible ni dans l'infra rouge aucun effet extincteur, aucune illumination fugace renforçant momentanément la fluorescence, aucune luminescence résiduelle de fluorescence. Des observations analogues ont été faites sur la leucophane de Brewig (fluorescence violette), le rubis lle Mladagascar (fluorescence rouge vif) la topaze (fluorescence jaune verdàtre), la calcite (fluorescence rougeàtre), le disthène (fluorescence rouge), le sel gemme (fluorescence bleue violette pàle), la strontianite (fluorescence rougeàtre), la willemite de New Jersey (fluorescence blanche), l'acide salicylique (fluorescence blanche), l'uranite (fluorescence verte), l'acétate d'urane (fluorescence verte), l'ambre de la Baltique (fluorescence vert pâle) colloïde.

D'autre part, Maurice Curie a constaté dans des conditions expérimentales très analogues l'absence d'effet extincteur avec la fluorescéine, l'esculine, les rhodamines B et $6 \mathbf{G}$, le platinocyanure de baryum, le nitrate d'urane, le sulfate d'urane et le potasse, le tungstate de calcium et la willemite. Nous avons obtenu avec ces corps des résultats identiques.

Interprétation physique. - Si l'on envisage l'ensemble des faits contenus dans cette étude on remarque d'une part la disparition de l'effet extincteur, de l'illumination fugace et de la luminescence résiduell e lorsque la persistance devient très brève, et d'autre part la connexité entre ces trois phénomènes. En particulier tout se passe comme si l'illumination fugace et la luminescence rémanente étaient deux modalités différentes d'un seul et même phénomène : la propriété que possède la matière de conserver à l'état potentel un résidu d'énergie lumineuse expulsé dans ce cas par l'apport des quanta infra-rouges antagonistes.

L'appareillage utilisé dans ce travail ayant été construit gràce à une subvention de l'Office National des Recherches, nous tenons à exprimer notre vive gratitude à M. le Professeur Langevin et à M. le Directeur de l'Enseignement supérieur.

(1) E. Gaviola. Z. f. Phys., 35 (1926), p. 748.

(2) F. Perrir. Polarisation de la lumière de fluorescence. Vie moyenne des molécules à l'état excité [J. de Phys, t. VII S. Vl, no 12, (1926), p. 390-401.] - R. Delorme et F. Perrin. Durée de fluorescence des sels d'uranyles solides et de leurs solutions. J. de Phys., S. VI, t. X, no ă (1929), p. 17i-186.]

$\left({ }^{3}\right)$ M. Gurie. Action des radiations rouges et infra-rouges sur les substances photoluminescentes $[J$. de Chimie Phys., t. 20, no 3 (1923), p. 294-296!. 